\title{
Implementación de un ciclo de mejora en el aula de la asignatura Francés Turístico
}

\section{Implementation of an improvement cycle in classroom of french as a foreign language in Tourism Context}

ADAMANTÍA ZERVA

ORCID: https://orcid.org/0000-0003-2883-7091

Universidad de Sevilla

Departamento de Filología Francesa

adamantía@us.es

Fecha de recepción: 04-06-2019

Fecha de aceptación: 16-06-2019

DOI: http://dx.doi.org/10.12795/9788447221912.023

Pp.: 541-560 


\section{RESUMEN}

El presente artículo resume los principales aspectos de la aplicación docente de un Ciclo de Mejora en el Aula (CIMA) en la asignatura de Francés Turístico, que se imparte en el segundo curso del Grado en Turismo. Se trata de una asignatura optativa y el número del alumnado asciende a 150 estudiantes, que se reparten en tres grupos. La enseñanza incluye contenidos teóricos así como prácticos y se emplea como metodología docente el enfoque comunicativo para el aprendizaje del Francés como lengua extranjera aplicado al sector turístico.

Palabras clave: Francés Turístico; Grado en Turismo; Docencia universitaria; Experimentación docente universitaria; Enfoque comunicativo.

\section{ABSTRACT}

This article summarizes the main aspects of the teaching application of an Improvement Cycle in Classroom (ICIC) in the subject of French as a Foreign Language in Tourism context which is taught in the second year of the Degree in Tourism. It is an optional subject and the number of students amounts to 150, divided into three groups. Teaching includes theoretical as well as practical content and the methodology applied is the communicative approach for teaching French as a foreign language in Tourism context.

Keywords: French as a Foreign Language in Tourism context; Degree in Tourism; University teaching; University teaching experimentation; Communicative approach.

Jornadas de Formación e Innovación Docente del Profesorado | № 2 (2019) Esta obra se distribuye con la licencia Creative Commons 


\section{Contextualización}

Los alumnos del Grado en Turismo cursan por primera vez en el segundo curso la asignatura optativa de Francés Turístico I cuyo contenido está orientado al aprendizaje de la terminología y las fórmulas de la lengua francesa que se emplean en el sector turístico. Para ello, se usa el manual didáctico titulado "Hôtellerie-restauration.com" (Corbeau et al., 2006) destinado a alumnos de un nivel inicial A1A2 (según el MCER) de Francés como lengua extranjera. Se trata de un libro de enseñanza de la lengua francesa en el contexto turístico dirigido a principiantes pero es necesario que los alumnos hayan cursado como mínimo 60 horas de clases de francés para que tengan unos conocimientos previos. En cada grupo, hay aproximadamente 45-55 alumnos cuyo nivel del francés es muy heterogéneo, un factor que dificulta la elaboración y aplicación de una puesta en práctica común.

\section{Diseño del Ciclo de Mejora en el Aula}

\section{Contenido}

El contenido de nuestra unidad didáctica es tratar los aspectos lingüísticos y sociolingüísticos relacionados con la gestión de reservas en un hotel. En el siguiente mapa de contenidos (Figura 1) hemos intentado resumir los principales a enseñar.

A nivel conceptual, lo que se enseña es reservar una habitación en un hotel y todas las destrezas lingüísticas relacionadas con esta acción comunicativa.

A nivel aptitudinal, se trata de adquirir competencias de índole gramatical, léxica y pragmática relacionadas con la tarea de realizar y gestionar una reserva en un hotel. 
A nivel actitudinal, se enseña una actitud de respeto haciendo hincapié en las diferencias socioculturales y adoptando una competencia intercultural que permite al alumno interactuar empleando la lengua francesa
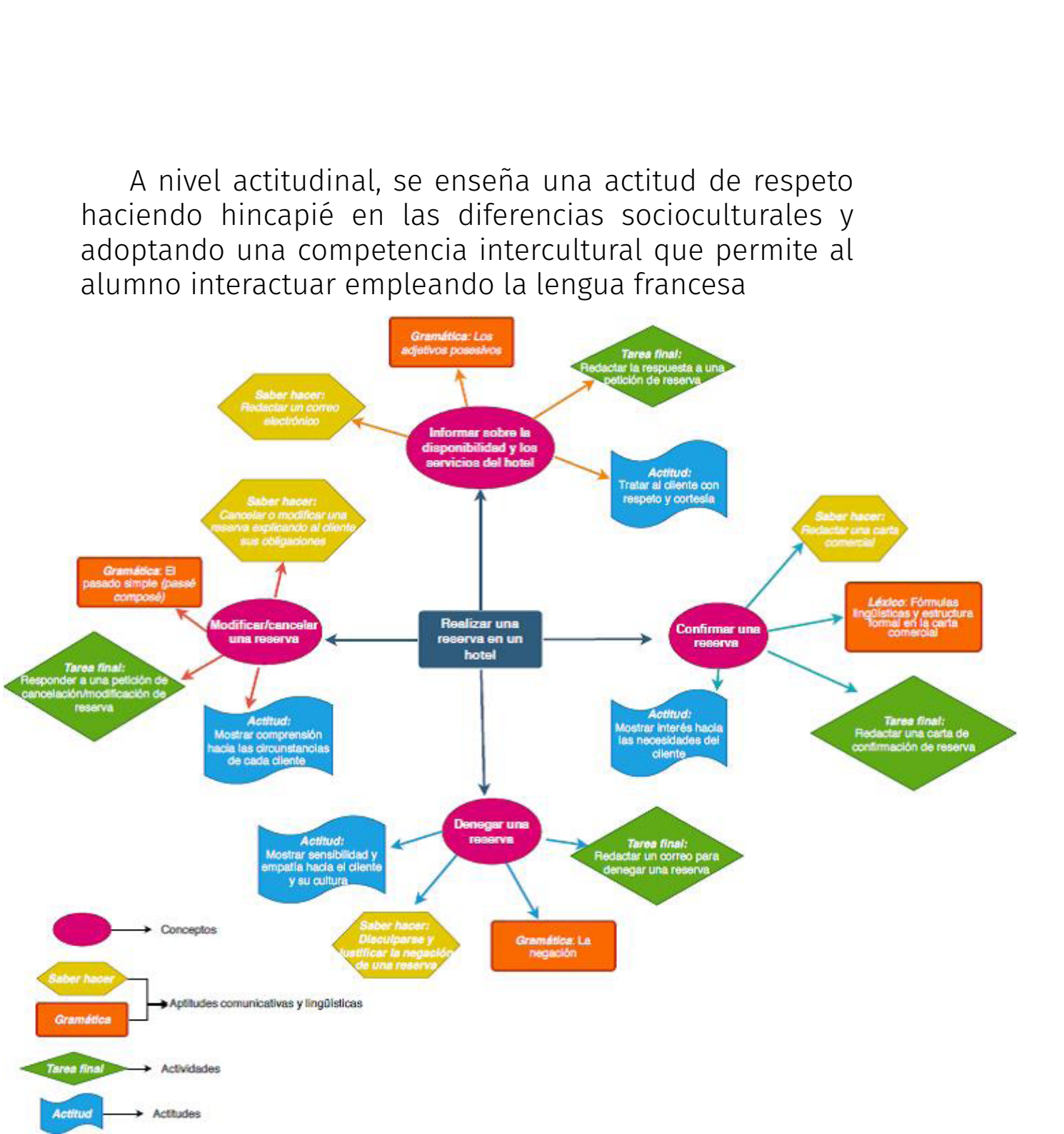

Figura 1. Mapa de contenidos.

\section{Objetivos y modelo metodológico}

El modelo metodológico que se emplea en la enseñanza de lenguas extranjeras desde hace aproximadamente treinta años es el método comunicativo (Richards y Rodgers, 1998), cuyo principio es centrarse en el alumno y sus necesidades aplicando una metodología basada en 
la acción para practicar la lengua en contexto real (a partir de situaciones simuladas, juegos de rol, etc). Sin embargo, la enseñanza de la gramática sigue desempeñando un papel importante. Breen (1987, pp. 85-87) realizó una descripción detallada de los objetivos y funcionamiento del "enfoque gramatical" que se basa fundamentalmente en el concepto de la lengua como un sistema. Desde esta perspectiva, se hace hincapié en los elementos que forman el sistema lingüístico y el proceso de aprendizaje se organiza según las destrezas. García Santa-Cecilia (2000, p. 65) señaló que este enfoque facilita al alumno la adquisición de las estructuras de una lengua extranjera. Los contenidos están organizados según su dificultad de forma que el aprendiz empieza por los elementos menos complejos hasta llegar a los estructuras más complejas. Desde esta perspectiva, nuestro modelo metodológico será el siguiente (Pregunta - Teoría - Práctica):

\section{$? \leftrightarrow \mathrm{T} \leftrightarrow \mathrm{P}$}

Comenzaremos por una pregunta general (Bain, 2007, p. 115) para activar los conocimientos lingüisticos y socioculturales que tienen los alumnos sobre su propia lengua y cultura y, a continuación, enseñaremos los contenidos lingüisticos en francés para realizar la tarea comunicativa propuesta, "Cómo realizar y gestionar la reserva de una habitación en un hotel".

Los objetivos para este ciclo de mejora en el aula son los siguientes:

1. Crear una pregunta inicial que ayudará a los alumnos a reflexionar sobre su propio proceso de aprendizaje desarrollando de esta manera estrategias metacognitivas y aplicando las estrategias de mediación entre su propia lengua y cultura y la lengua-cultura meta.

2. Dedicar más tiempo a la práctica de la expresión oral. 
3. Aumentar la participación en clase y la colaboración entre los estudiantes.

4. Asimilar los contenidos gramaticales previstos así como las formas lingüísticas para redactar una carta formal comercial.

\section{Evaluación de los conocimientos iniciales de los alumnos}

Los cuestionarios iniciales son un recurso muy útil para descubrir los conocimientos previos de los alumnos de índole lingüística, dado que si no se dominan algunas estructuras previas y más simples es muy dificil llegar a las más complejas. En el cuestionario inicial, hemos incluido diez preguntas breves de elección múltiple para evaluar esos conocimientos iniciales de los alumnos sobre cuestiones lingüísticas clave para el aprendizaje de los contenidos de nuestro ciclo de mejora (Tabla 1).

Tabla 1. Cuestionario inicial.

I. Prueba de conocimientos previos (pre-actividad)

Testez- vous en français- Choisissez la bonne réponse:

1. Où est ma .................?

a. chambre b. hôtel $\mathbf{c}$. restaurant $\mathbf{d}$. assiette

2. Elle n'aime pas ..............hôtels modernes.

a. l' b. les c. des d. de

3. Nous allons France.

a. à b. au c. aux d. en

4. Je prends mon dîner á huit heures ...........demie.

a. et b. plus c. moins d. de

5. Où ..-vous?

a. parlez b. faites c. avez d. allez 6. ...comment vas-tu?

a. Au revoir b. Merci c. Pardon d. Bonjour

Jornadas de Formación e Innovación Docente del Profesorado | № 2 (2019) Esta obra se distribuye con la licencia Creative Commons Reconocimiento-NoComercial-SinObraDerivada 4.0 Internacional (CC BY-NC-ND 4.0.) 
7. Le chiffre 96 se lit:

a. soixante-six b. quatre- vingt-six c. soixante-seize $\mathbf{d}$.

quatre-vingt-seize

8. Un client se présente à la réception. Je dis:

a. Salut b. Bonjour, comment vas-tu?

c. Bonjour, comment allez-vous? d. Bonjour, Monsieur

9. Un client part. Je dis:

a. Au revoir b. Au revoir, monsieur c. Salut d. Adieu

10. Je propose mes services à un client. Je dis:
a. C'est pour quoi?
b. Qu'est-ce que vous voulez?
c. Qu'est-ce que tu veux?
d. Je peux vous aider?

\section{Secuencia de actividades programada}

La unidad didáctica, como ya habíamos mencionado, tenía como objetivo la adquisición de la competencia comunicativa de "Cómo realizar y gestionar la reserva de una habitación en un hotel". Para abordar ese objetivo se ha programado una secuencia de actividades que pretende trabajar la situación planteada desde la lógica del modelo didáctico expuesto.

Con respecto a las estrategias docentes que aplicaremos para conseguir los objetivos de este ciclo de mejora, nos gustaría mencionar los siguientes aspectos fundamentales:

- Se empezará por una pregunta para que los alumnos practiquen la competencia de mediación, es decir, mediar entre su cultura y la cultura meta.

- Se dedicará tiempo a la expresión oral, más de lo habitual, pero todavía es mucho menos del tiempo dedicado a la realización de ejercicios de tipo escrito.

- Por último, en las tareas que se realizarán en casa, los alumnos practicarán las competencias necesarias para responder por escrito a una petición de reserva.

En la Tabla 2 se recoge el diseño básico de la secuencia de actividades en cada sesión de clase. 


\section{Sesión 1: Realizar una reserva por correof electrónico}

I. Primera actividad: Se introduce el contenido con una pregunta para que los alumnos reflexionen sobre el proceso a seguir para realizar una reserva en un hotel. Cuando se hayan mencionado todas las etapas del proceso de reserva, se ordenan según la estructura fijada en la lengua francesa.

II. Segunda actividad: Se realiza un ejercicio auditivo para ver cómo responder a la petición de una reserva por correo electrónico. El ejercicio consiste en poner en orden las distintas partes de la carta formal de solicitud de reserva.

III. Tercera actividad: Ejercicio de comprensión escrita que consiste en leer un correo electrónico de petición de reserva y contestar a ocho frases eligiendo verdadero o falso. Los alumnos trabajan en grupos de dos o tres e intentan encontrar las preguntas que faltan.

IV. Cuarta actividad: Se trata de un ejercicio de competencia léxica en el que hay que completar las expresiones relacionadas con la redacción de un correo electrónico formal respondiendo a una solicitud de reserva.

V. Quinta actividad: Practicar los adjetivos posesivos mediante un ejercicio escrito de forma inductiva completando los posesivos que faltan en cuatro frases.

VI. Sexta actividad: Como última actividad, se practica la competencia de expresión oral. Los alumnos se dividen en dos grupos. El primer grupo redacta una solicitud de reserva y el segundo grupo responde. A continuación, practicamos de forma oral el mismo ejercicio.

\section{Sesión 2: Confirmar una reserva por escrito}

I. Primera actividad: Se introduce el contenido invitando a los alumnos a reflexionar sobre las expresiones de correspondencia escrita en español. A continuación, se presentan los términos clave en una carta formal como remitente, destinatario, asunto, fórmula de saludo/despedida, firma, etc.

II. Segunda actividad: Se realiza un ejercicio de comprensión escrita sobre los contenidos tratados en el ejercicio anterior.

Jornadas de Formación e Innovación Docente del Profesorado | № 2 (2019) Esta obra se distribuye con la licencia Creative Commons Reconocimiento-NoComercial-SinObraDerivada Internacional (CC BY-NC-ND 4.0.) 
III. Tercera actividad: Se introduce un ejercicio de comprensión escrita y de competencia léxica, dado que consiste en completar las frases con las palabras que faltan.

IV. Cuarta actividad: Se practica el vocabulario mediante un ejercicio para corregir las expresiones relacionadas con la redacción de un correo electrónico formal que tiene como asunto responder a una solicitud de reserva. En el presente ejercicio, los alumnos tienen que sustituir las frases o palabras erróneas por las apropiadas en una carta formal de confirmación de reserva. Los aspectos a tratar son de contenido léxico, gramatical y estilístico.

V. Quinta actividad: Se trata de un ejercicio de comprensión oral relacionado con el ejercicio anterior. Se escucha al recepcionista que corrige las erratas de la carta de confirmación de reserva. De esta manera los alumnos comprueban sus respuestas y practican la comprensión oral.

\section{Sesión 3: Denegar una reserva}

I. Primera actividad: Se introduce el contenido invitando a los alumnos a reflexionar sobre las expresiones que se pueden emplear para denegar una reserva a un cliente. A continuación, se presentan los términos clave y la estructura de la interacción oral o escrita de este tipo: lo siento pero el hotel está completo, proponer una solución, etc.

II. Segunda actividad: Se realiza un ejercicio de comprensión oral sobre los contenidos tratados en el ejercicio anterior. Se trata de asociar seis viñetas con seis diálogos.

III. Tercera actividad: Ejercicio de producción oral. Se presentan tres situaciones simuladas y los alumnos tienen que negar la reserva siguiendo la estructura que se explicó al principio de la clase. Se realiza en parejas y los resultados son muy buenos.

IV. Cuarta actividad: Se practica la comprensión escrita: se leen dos mensajes y hay que responder a cuatro preguntas sobre el destinatario, el asunto del mensaje, el remitente, el problema y la respuesta. 
V. Quinta actividad: Se presenta un contenido gramatical: la negación. En primer lugar, se presenta la teoría (formas de la frase negativa) $y$, en segundo lugar, se practican los aspectos teóricos mediante ejercicios.

VI. Sexta actividad: Se realiza un ejercicio de producción oral y escrita: el recepcionista del hotel recibe un correo con una petición de ampliación de estancia. Debe denegarla y proponer una solución. Se interpreta la escena en parejas en clase. Como tarea para practicar en casa, se realiza el mismo ejercicio pero se entrega por escrito en la siguiente sesión.

\section{Sesión 4: Modificar/cancelar una reserva}

I. Primera actividad: Se introduce el contenido invitando a los alumnos a reflexionar sobre las expresiones que se pueden emplear para modificar/anular una reserva de un cliente. A continuación, se presentan los términos clave y la estructura de la interacción oral y escrita de este tipo.

II. Segunda actividad: Se realiza un ejercicio de comprensión oral sobre los contenidos tratados en el ejercicio anterior. Se trata de rellenar una ficha de modificación de reserva con los cambios solicitados.

III. Tercera actividad: Se practica la comprensión y producción oral. En primer lugar, los alumnos escuchan un diálogo con un cliente que solicita un cambio y realizan un ejercicio sobre afirmaciones verdaderas o falsas. En segundo lugar, tienen que hacer un juego de rol: se les presentan tres situaciones simuladas para negar la reserva y eligen una para interpretar la escena y presentarla en clase.. El ejercicio se realiza en parejas (cliente-recepcionista) y los resultados son muy buenos.

IV. Cuarta actividad: Se presenta un contenido gramatical: la forma y el empleo del pasado simple de forma deductiva. En primer lugar se presenta la teoría (forma-uso) y en segundo lugar se practican los aspectos teóricos mediante ejercicios.

V. Quinta actividad: Se trata de un ejercicio de producción escrita: el recepcionista del hotel recibe un correo con una petición de anulación de reserva. Debe contestar explicando la política de reserva. 


\section{Evaluación del aprendizaje del alumno}

Como parte del proceso de evaluación, emplearemos el siguiente cuestionario final (Tabla 3), compuesto de cuatro preguntas de respuesta abierta sobre las principales tareas comunicativas relacionadas con el contenido que se impartirá: realizar y gestionar la reserva en un hotel:

Tabla 3. Cuestionario final

UNITÉ 2: RESERVATION

1. Vous êtes réceptionniste à l' hôtel de la Paix. Vous répondez au téléphone. Que dites-vous?

2. Á la fin d’un courrier électronique, pour saluer le destinataire, vous écrivez :

3. Vous êtes réceptionniste. Un client téléphone pour réserver une chambre mais vous refusez cette réservation parce que l' hôtel est complet. Que dites- vous?

4. Vous prenez la réservation d’une chambre. Quelles questions posez-vous pour connaître les dates d’arrivée et de départ d’un client?

\section{Aplicación del Ciclo de Mejora en el Aula}

\section{Relato resumido de las sesiones}

En la primera sesión, aplicamos la metodología docente innovadora que aprendimos en el Curso General de Docencia Universitaria y planteamos una pregunta a los estudiantes sobre el proceso de la realización de una reserva. Les invitamos a reflexionar sobre la actuación de un recepcionista cuando recibe la petición de una reserva. Observamos que los estudiantes se esfuerzan para cambiar de perspectiva, porque hasta entonces reaccionaban 
como clientes, no como profesionales del turismo. Gracias a esta primera puesta en práctica, desarrollaron su competencia de mediación entre su cultura y la cultura meta.

En la segunda sesión, comenzamos la clase empleando la misma estrategia y planteamos una pregunta a los alumnos sobre la estructura de una carta formal en su lengua materna con la intención de que nos sirviese de esquema para basar los nuevos contenidos en la lengua francesa. Para nuestra sorpresa, ningún alumno estaba familiarizado con el formato de una carta comercial en español. Les invitamos a investigar empleando su teléfono móvil y, finalmente, se consiguió elaborar un guion para la redacción de una carta formal en español. A continuación, dimos las pautas para realizar la misma tarea en francés en el contexto turístico. Esta primera actividad suscitó el interés de los alumnos y motivó una reflexión muy enriquecedora sobre los aspectos diferenciadores de las dos culturas y lenguas. No obstante, el tiempo que se dedicó para completar la actividad superó el que habíamos estimado inicialmente.

En la tercera sesión, nuestro objetivo fue abordar algunos aspectos de indole léxica y gramatical. Para ello, nos centramos en las expresiones que se emplean para denegar una reserva. Hicimos hincapié en el registro formal que se usa en cualquier tipo de interacción entre el recepcionista y el cliente. La sociedad francesa es más jerarquizada que la española y es necesario que los aprendices sean conscientes de que la formalidad y la cortesía son dos aspectos imprescindibles en el sector turístico. Al final de esta sesión, realizamos un juego de rol en parejas y el resultado fue muy satisfactorio. Los alumnos participaron con entusiasmo y se esforzaron para simular una interacción en condiciones reales.

Por último, en la cuarta sesión, la puesta en práctica de la unidad didáctica se desarrolló con mayor éxito. 
Observamos que los estudiantes estaban familiarizados con la metodología aplicada (Pregunta-Teoría-Práctica) y respondían mucho mejor a las preguntas planteadas. Asimismo, se constató una mayor participación en clase y un ambiente de colaboración entre los estudiantes. Nos dimos cuenta de que la realización de actividades en parejas había mejorado las relaciones entre los estudiantes, un hecho que favorece la dinámica de la clase y, por consiguiente, crea un ambiente más propicio para el aprendizaje cooperativo. En la última sesión, dedicamos más tiempo a la comprensión oral. Además, abordamos un contenido gramatical muy importante: el pasado simple. La metodología que se empleó fue deductiva y, a continuación, se practicó la forma y el uso a través de ejercicios. En este punto, hemos de decir, que el resultado fue muy satisfactorio aunque la evaluación final demostraría si el contenido gramatical se habría asimilado. Por último, concluimos la sesión con un juego de rol para poner en práctica los contenidos gramaticales y discursivos de la acción comunicativa de modificar/anular una reserva. La realización de los diálogos tuvo un resultado satisfactorio aunque la mayoría de los estudiantes no siguieron el guion inicial de la actividad propuesta sino que improvisaron sin cometer errores, un hecho que valoramos positivamente.

\section{Evaluación del aprendizaje de los estudiantes}

Como ya hemos mencionado, consideramos que los cuestionarios iniciales son un recurso muy útil para explorar y aproximarnos a los esquemas mentales de los alumnos (Rivero y Porlán, 2017) y, en nuestro caso, nos ayudaron a descubrir sus conocimientos previos. Sin embargo, el aprendizaje de una lengua extranjera se basa en adquirir conocimientos lingüísticos y si no se dominan algunas estructuras previas y más simples es muy dificil llegar a las más complejas. En este sentido, aplicar el mismo modelo de cuestionario inicial y final no es conveniente en 
nuestra materia porque, al tratarse de contenidos lingüísticos, los alumnos no pueden descubrirlos. Por tanto, hemos tenido que crear dos cuestionarios no idénticos pero sí complementarios que siguen el esquema de una escalera empezando por las preguntas más simples hasta las más complejas.

Con respecto a los criterios de evaluación, hemos empleado un criterio holístico basado en el resultado de la evaluación de sus conocimientos a partir del estudio comparativo de los dos cuestionarios teniendo en cuenta tres valores:

a) El resultado del cuestionario inicial que indica el nivel de partida y los conocimientos previos.

b) El resultado del cuestionario final, puesto que demuestra si los alumnos han asimilado los contenidos enseñados.

c) La comparación de los resultados de los dos cuestionarios.

Este último valor es muy importante para evaluar el progreso de cada alumno. Como habíamos visto al principio, el primer cuestionario se basaba en evaluar los conocimientos iniciales en la lengua francesa. Un alumno que tiene un buen nivel de francés se espera que aprenda a un nivel muy satisfactorio los nuevos contenidos. Sin embargo, si un alumno que tiene un nivel medio consigue un resultado muy bueno, habrá que evaluar más su progreso. Por el contrario, un alumno que tiene un resultado bueno o medio en el cuestionario inicial y no consigue obtener un resultado de más de $50 \%$ en el cuestionario final, consideramos que su progreso es poco satisfactorio. Si el porcentaje presenta un descenso de más del 30\% del resultado del primer cuestionario, entonces su progreso es considerado insuficiente. Por consiguiente, distinguimos cuatro niveles-escalones con respecto al progreso (Tabla 4).

Jornadas de Formación e Innovación Docente del Profesorado | № 2 (2019) Esta obra se distribuye con la licencia Creative Commons 
Tabla 4. Descriptores de los escalones de progreso.

\begin{tabular}{|c|c|}
\hline Escalón & Descriptores \\
\hline $\begin{array}{l}\text { Escalón 4: } \\
\text { Muy } \\
\text { satisfactorio }\end{array}$ & $\begin{array}{l}\text { - Los resultados del } 2 \text { - cuestionario son muy satisfac- } \\
\text { torios, resultado superior al } 85 \% \text {, algo que demues- } \\
\text { tra que el alumno ha adquirido los conocimientos } \\
\text { impartidos. } \\
\text { - El resultado del segundo cuestionario es superior al } \\
\text { del primero y la diferencia es superior al } 30 \% \text {. }\end{array}$ \\
\hline $\begin{array}{l}\text { Escalón 3: } \\
\text { Satisfactorio }\end{array}$ & $\begin{array}{l}\text { - El resultado del } 20 \text { cuestionario es satisfactorio (su- } \\
\text { perior al } 75 \% \text { ), lo que demuestra haber adquirido los } \\
\text { conocimientos impartidos. } \\
\text { - La diferencia entre los resultados de los dos cuestio- } \\
\text { narios varía mínimamente }(10 \%) \text {. }\end{array}$ \\
\hline $\begin{array}{l}\text { Escalón 2: } \\
\text { Poco } \\
\text { satisfactorio }\end{array}$ & $\begin{array}{l}\text { - Tiene unos conocimientos de nivel básico del francés } \\
\text { pero no ha alcanzado los objetivos del 2o cuestiona- } \\
\text { rio (resultado < 30\%). } \\
\text { - El resultado del segundo cuestionario es inferior al } \\
\text { del primero pero la diferencia es inferior al 30\%. }\end{array}$ \\
\hline $\begin{array}{l}\text { Escalón 1: } \\
\text { Insuficiente }\end{array}$ & $\begin{array}{l}\text { - Tiene unos conocimientos de nivel básico de francés } \\
\text { (resultado del 1o cuestionario entre } 60-65 \% \text { ) pero no } \\
\text { ha alcanzado los objetivos del 20 cuestionario (resul- } \\
\text { tados }<30 \% \text { sobre el total). } \\
\text { - El resultado del segundo cuestionario es inferior al } \\
\text { del primero y la diferencia es superior al } 30 \% \text {. }\end{array}$ \\
\hline
\end{tabular}

En las Tablas 5 y 6 y en las Figuras 2 y 3 se sintetiza el estudio comparativo de los resultados de los cuestionarios inicial y final por alumno, mostrándose la evolución del progreso individual. El corpus que hemos empleado para realizar el análisis comparativo de los dos cuestionarios se compone de la evaluación de ocho alumnos en total. En la Tabla 5, en la primera columna, aparece la relación de los alumnos mientras que en la segunda y tercera columna aparecen los resultados obtenidos en cada cuestionario. 
La última columna refleja la evaluación del progreso realizado según los escalones descritos en la Tabla 4 que corresponden a cuatro niveles de progreso: insuficiente / poco satisfactorio / satisfactorio / muy satisfactorio.

Tabla 5. Análisis comparativo de los resultados de los cuestionarios por alumno.

\begin{tabular}{|l|l|l|l|}
\hline Alumno & Cuestionario inicial & Cuestionario final & \multicolumn{1}{c|}{ Evaluación } \\
\hline Alumno A & $90 \%$ & $83 \%$ & satisfactorio \\
\hline Alumno B & $70 \%$ & $87,5 \%$ & muy satisfactorio \\
\hline Alumno C & $70 \%$ & $91,6 \%$ & muy satisfactorio \\
\hline Alumno D & $80 \%$ & $87,5 \%$ & muy satisfactorio \\
\hline Alumno E & $90 \%$ & $41,6 \%$ & insuficiente \\
\hline Alumno F & $70 \%$ & $45,8 \%$ & poco satisfactorio \\
\hline Alumno G & $60 \%$ & $21,1 \%$ & insuficiente \\
\hline Alumno H & $80 \%$ & $79 \%$ & satisfactorio \\
\hline
\end{tabular}

En la Tabla 6 se ilustra el porcentaje de alumnos que alcanzó cada escalón y en la Figura 2 aparece la representación gráfica en forma de escalera de progreso.

Tabla 6. Porcentajes de alumnos por escalones en la evaluación final.

\begin{tabular}{|l|l|l|l|}
\hline \multicolumn{1}{|c|}{ Escalón } & \multicolumn{2}{|c|}{ Evaluación del progreso } & \\
\hline & $\begin{array}{c}\text { Diferencia del resultado } \\
\text { de los cuestionarios }\end{array}$ & $\begin{array}{c}\text { № de } \\
\text { alumnos }\end{array}$ & $\begin{array}{c}\text { Porcentaje de } \\
\text { alumnos }\end{array}$ \\
\hline Escalón 4 & $1 \underline{0}<2$ o & 3 & $37,5 \%$ \\
\hline Escalón 3 & $1 \underline{0}=2 \underline{0}$ & 2 & $25 \%$ \\
\hline Escalón 2 & $1 \underline{0}>2 \underline{0}$ & 1 & $12,5 \%$ \\
\hline Escalón 1 & $1 \underline{0}>2 \underline{0}+30 \%$ & 2 & $25 \%$ \\
\hline
\end{tabular}

Jornadas de Formación e Innovación Docente del Profesorado | № 2 (2019) Esta obra se distribuye con la licencia Creative Commons Reconocimiento-NoComercial-SinObraDerivada Internacional (CC BY-NC-ND 4.0.) 


\section{Escalón 1}

Figura 2. Evaluación final del progreso.

A continuación, en la Figura 3, se muestran de forma conjunta los resultados de los dos cuestionarios así como el progreso alcanzado que surge de la comparación entre ambos según el porcentaje de alumnos en cada escalón (E1-E2-E3-E4).

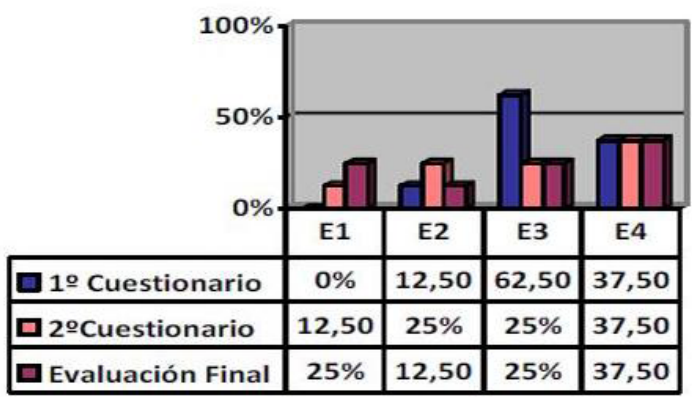

\begin{tabular}{|l|}
\hline $1 \%$ Cuestionario \\
$\square 2 \%$ Cuestionario \\
$\square$ Evaluación Final
\end{tabular}

Figura 3. Representación gráfica de los resultados conjuntos según los escalones.

A modo de conclusión, se podría afirmar que los cuestionarios inicial y final nos han sido de gran utilidad para el diseño y valoración de nuestro ciclo de mejora. Los resultados del primer cuestionario nos hicieron ver que el nivel de conocimientos previos de nuestros alumnos 
equivalía al tercer escalón $(62,5 \%)$ y, por consiguiente, teníamos alumnos con un nivel satisfactorio de francés. Asimismo, un 37,5\% tenía un alto nivel (escalón 4), un hecho que facilitaría nuestra actividad docente.

Según los resultados del cuestionario final, el porcentaje de alumnos de alto nivel que pertenecían al cuarto escalón se mantuvo y llegó a los objetivos docentes establecidos, un hecho que nos da satisfacción. Así pues, desde una perspectiva pedagógica se podría pensar que se trata de un grupo de alumnos muy motivados que estudian mucho para alcanzar sus objetivos. Por consiguiente, el éxito o no de nuestra actividad docente no se puede evaluar a partir del resultado obtenido por estos alumnos ideales. El 25\% de los alumnos llegó al tercer escalón y alcanzó un nivel satisfactorio de los contenidos enseñados. No obstante, se trata de un porcentaje que se mantuvo estable con respecto a su rendimiento en el primer y segundo cuestionario. Asimismo, un 12,5\% de los alumnos tuvo un leve descenso en su rendimiento en comparación a los resultados del cuestionario inicial y no llegó a alcanzar los objetivos consiguiendo un nivel poco satisfactorio. Por último, un 25\% empeoró notablemente su rendimiento y no llegó a los conocimientos mínimos exigidos. Sería interesante indagar sobre las causas para que encontrásemos una solución.

\section{Evaluación del diseño puesto en práctica}

\section{Aspectos a mantener y cambios a introducir}

Después de la puesta en práctica del segundo ciclo de mejora y partiendo de los resultados de la evaluación final de los estudiantes hemos podido llegar a unas valoraciones generales sobre los resultados de nuestra unidad didáctica (autoevaluación de nuestra docencia): 
- En primer lugar, como innovación en nuestra práctica docente, empezamos por una pregunta en cada sesión para que los alumnos reflexionasen y activasen la competencia de mediación, es decir, mediar entre su cultura y la cultura meta. Sin embargo, es cierto que nos hubiese gustado haber encontrado una forma más deductiva para introducir el contenido didáctico de "cómo realizar y gestionar una reserva en un hotel".

- En segundo lugar, se ha dedicado más tiempo de lo habitual a la expresión oral, pero todavía es mucho menos del tiempo dedicado a la realización de ejercicios de tipo escrito. Convendría mejorar este aspecto en un futuro.

- Por último, en este segundo ciclo de mejora, no disponíamos todavía de los resultados de las tareas que se realizaron en casa por escrito para comprobar si los objetivos se han alcanzado en todas las competencias. No obstante, hemos podido observar que ha habido mayor participación en clase debido, entre otros factores, al hecho de que el ritmo fue más lento y se aclaraban las dudas a nivel individual, un hecho que permitió a todos los alumnos participar con la confianza de que lo que decían era correcto.

En líneas generales, se podría afirmar que el resultado de este ciclo de mejora ha sido satisfactorio en cuanto a la metodología aplicada, a la puesta en práctica, asi como al sistema de evaluación. Sin embargo, convendría mejorar las estrategias para practicar la lengua oral en clase.

Jornadas de Formación e Innovación Docente del Profesorado | № 2 (2019) Esta obra se distribuye con la licencia Creative Commons 


\section{Referencias bibliográficas}

Bain, K. (2007). Lo que hacen los mejores profesores universitarios. Valencia: Publicacions de la Universitat de València.

Breen, M. P. (1987): Contemporary Paradigms in Syllabus Design- Part I., Language Teaching, 20(2), 81-92.

Corbeau, S. et al. (2006). Hôtellerie-restauration.com. Français professionnel. Paris: Clé International.

Finkel, D. (2008). Dar clase con la boca cerrada. Valencia: Publicacions de la Universitat de València.

García Santa-Cecilia, A. (2000). Cómo se diseña un curso de lengua extranjera. Madrid: Arco Libros.

Richards, J. C. y Rodgers, T. S. (2003[1998]). Enfoques y métodos en la enseñanza de idiomas. Traducción española de José M. Castrillo y María Condor. Cambridge: Cambridge University Press, segunda edición.

Rivero, A. y R. Porlán (2017). La evaluación en la enseñanza universitaria. En R. Porlán (coord.), Enseñanza universitaria. Cómo mejorarla (pp. 73-91). Madrid: Morata.

Jornadas de Formación e Innovación Docente del Profesorado | № 2 (2019) Esta obra se distribuye con la licencia Creative Commons Reconocimiento-NoComercial-SinObraDerivada 4.0 Internacional (CC BY-NC-ND 4.0.) 